\title{
Iron deficiency biomarkers prescription in the French pediatric population: evolution in the last 10 years
}

Anne-Sylvia Sacri ${ }^{1,2,3}$, Nhung Trinh ${ }^{1}$, Mariane de Montalembert ${ }^{1,2}$, Amandine Ganon ${ }^{4}$, Laurent Gouya ${ }^{5}$, Martin Chalumeau ${ }^{1,2}$ ${ }^{1}$ INSERM UMR1153, Centre de Recherche en Épidémiologie et Statistique Sorbonne Paris Cité (CRESS), équipe Épidémiologie Périnatale, Obstétricale et Pédiatrique (ÉPOPé), Labex GR-Ex, Université Paris Descartes, Paris, France; ${ }^{2}$ Department of General Pediatrics and Pediatric Infectious Diseases, Necker-Enfants malades hospital, AP-HP, Paris, France; ${ }^{3}$ Université Paris Diderot, Sorbonne Paris Cité; Ambulatoire (AFPA); ${ }^{4}$ Laboratoire CERBA, Saint-Ouen l'Aumône, France; ${ }^{5}$ Centre de recherche sur l'inflammation, INSERM UMR 1149, Université Paris Diderot ; ERL CNRS 8252, Faculté de Médecine site Bichat, Paris, France

\section{Background}

- Accurate diagnosis of iron deficiency (ID) in infants is of paramount importance given its long term consequences and thus requires the use of adequate biomarkers among the numerous available ones $[1,2]$

- In France, recommendations regarding which biomarkers should be used for ID diagnosis have been updated in 2011 by the French National Authority for Health (HAS) [3] with the two following main messages: the recommended use of serum ferritin (SF) and the discouraged use of serum iron (SI) alone

- We aimed at studying the prescription of ID biomarkers in children in France and the impact of the 2011 national prescription guidelines for ID diagnosis

\section{Methods}

- We used the Echantillon Généraliste des Bénéficiaires (EGB) a national administrative database that collects all ambulatory medical acts (including biological tests) reimbursed by the national public social welfare for a random sample of $1 \%$ of French citizen

- We extracted data from 2006 to 2016 for all reimbursed ID biomarkers for patients < 15 years old

- We described the prescriptions' structure and its evolution

- Using an interrupted-time series approach, we evaluated the impact of the two main messages of the 2011 guidelines

\section{Results}

- During the study period, iron biomarkers were prescribed at least once to $3.9 \%$ of the pediatric population

- We observed an increase in the share of SF among iron biomarkers from $54.7 \%$ to $60.1 \%$ and a decrease in $\mathrm{SI}$ one from $40.0 \%$ to $31.8 \%$ (Figure 1)

- The 2011 guidelines had no significant impact on the trend of SF prescriptions nor SI prescriptions in comparison with the expected trend without intervention $(p=0.10)$ (Figure 2)

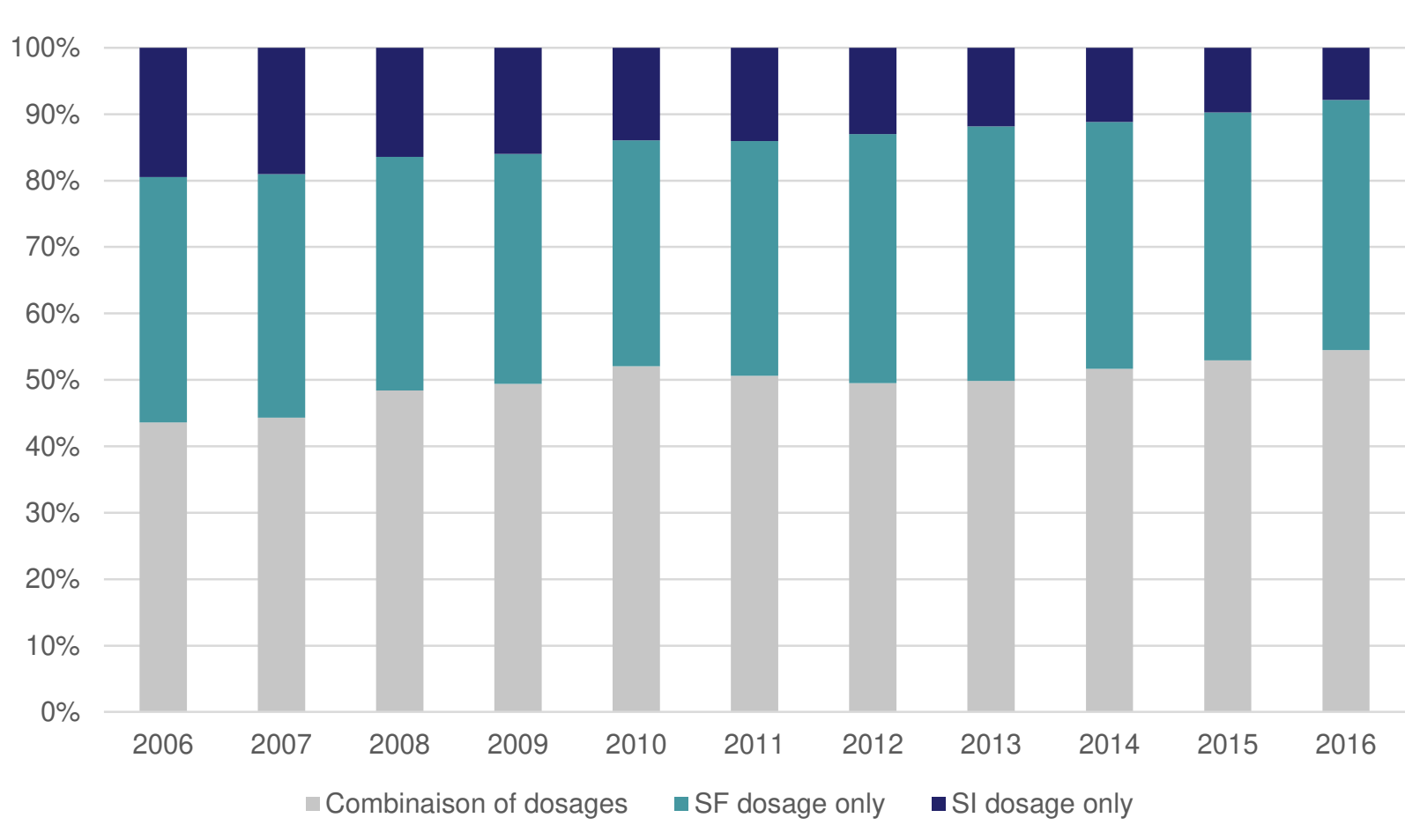

Figure 1. Structure of prescriptions

Figure 2. Impact of 2011 guidelines on prescriptions of SF dosage only and SI dosage only: interrupted time serie analysis

Grey zone: wash-out period for guidelines diffusion estimated from march 2011 to february 2012

A-B segment: prescriptions rates before intervention

C-E segment: prescriptions rates observed after intervention

D-F segment: prescriptions rates expected without intervention

Impact of guidelines: relative change in prescriptions rates estimated by $(E-F) / F$

\section{Conclusion}

- ID biomarkers prescription in children evolved slowly in the last decade towards a more frequent use of recommended biomarkers

- There was no observable impact of the 2011 guidelines on the pediatric population

Références: 1. Baker Pediatrics 2010, 2. Domellof J Pediatr Gastroenterol Nutr 2014, 3. [Choice of iron metabolism biomarkers for diagnostic of iron deficiency] HAS 2011 at www.has-sante.fr

COI and Funding: DGOS PHRC regional AOR14053, Secteur français des Aliments de l'Enfance, Université Paris Diderot Sorbonne Paris cité 